Les théories d'apprentissage et méthodes d'enseignement des langues étrangères : revue de littérature

\author{
Asma Nifaoui ${ }^{\mathrm{a} *}$ \\ ${ }^{a}$ Université Mohammed V, Rabat, Morocco \\ *nifaouiasma@gmail.com
}




\section{Les théories d'apprentissage et méthodes d'enseignement des langues étrangères : revue de littérature}

\section{Résumé}

L'objectif de cet article est de donner un aperçu historique sur les courants d'apprentissage qui ont marqué plusieurs domaines ces deux derniers siècles, et dont les méthodes d'enseignement des langues étrangères se sont largement inspirées. Enseigner et apprendre une langue étrangère a toujours été la préoccupation majeure des responsables exerçant dans le secteur éducatif. Trouver une méthode unique, parfaite et convenable à l'ensemble des apprenants était le centre d'intérêt de la plupart des chercheurs tous profils confondus, linguistes, pédagogues ou formateurs. Trois grandes théories d'apprentissage ont réellement bouleversé le domaine de l'éducation et spécifiquement le champ de l'acquisition des langues étrangères. Ces trois courants sont respectivement le behaviorisme, le cognitivisme et le constructivisme. Chaque courant avait ses partisans et ses détracteurs selon le progrès réalisé au terme de leurs travaux de recherche. Les méthodes qui ont découlé de ces trois théories d'apprentissage ont également connu des hauts et des bas selon le degré de l'atteinte des objectifs escomptés. Au cours de la présente étude, nous projetons de contourner, dans un ordre chronologique, les différentes méthodes et approches, provenant des trois courants, et pratiquées dans l'enseignement des langues étrangères. Notre attention sera focalisée sur l'étude de la méthode audio-orale, la méthode audiovisuelle, l'approche communicative et l'approche par compétences. Nous proposons aussi, dans cet article, une discussion dont l'intérêt est d'élucider les circonstances qui ont mené à la réussite ou à l'échec de telle ou telle méthode. Nous nous arrêterons également sur les réussites et les limites de chacune de ces méthodes. La conclusion fera l'objet d'une réflexion sur l'ensemble des points abordés dans l'article tout en proposant une ouverture sur d'autres solutions pouvant améliorer l'enseignement/apprentissage des langues étrangères.

Mots clés : théories d'apprentissage, méthodes d'enseignement des langues étrangères, behaviorisme, cognitivisme, constructivisme, approche communicative, l'approche par compétences. 


\title{
Theories of learning and methods of teaching foreign languages: Literature review
}

\begin{abstract}
The objective of this article is to provide a historical overview of the learning trends that have marked several fields over the past two centuries, and from which the methods of teaching foreign languages have been largely inspired. Teaching and learning a foreign language has always been a major concern of officials working in the education sector. Finding a unique method, perfect and suitable for all learners was the center of interest of most researchers of all backgrounds, linguists, educators or trainers. Three major theories of learning have really changed the field of education and specifically the field of foreign language acquisition. These three currents are respectively behaviorism, cognitivism and constructivism. Each current had its supporters and detractors depending on the progress made at the end of their research. The methods that emerged from these three learning theories also had their ups and downs depending on the degree to which the desired objectives were achieved. In the course of this study, we plan to bypass, in chronological order, the different methods and approaches, coming from the three streams, and practiced in the teaching of foreign languages. Our attention will be focused on the study of the audio-oral method, the audio-visual method, the communicative approach and the skills-based approach. We also offer, in this article, a discussion whose interest is to elucidate the circumstances which led to the success or failure of a particular method. We will also focus on the successes and limitations of each of these methods. The conclusion will be the subject of a reflection on all the points covered in the article while offering an opening on other solutions that can improve the teaching / learning of foreign languages.
\end{abstract}

Keywords: learning theories, foreign language teaching methods, behaviorism, cognitivism, constructivism, communicative approach, competency-based approach.

\section{Introduction}

L'intérêt de cet article est de proposer une analyse des principaux courants de 
l'apprentissage (behaviorisme, cognitivisme, constructivisme) ainsi que les méthodes d'enseignement qui en ont découlé. D'un côté, le rôle des théories est de fournir un cadre référentiel pour expliquer des phénomènes observés dans l'environnement, de l'autre côté, les théories servent également de ponts entre la recherche et les pratiques éducatives dont la fonction est d'organiser et traduire les résultats de la recherche tout en procédant à une opération d'adaptation entre les deux. Selon Schunk (2012), les théories de l'apprentissage et le domaine de l'éducation sont souvent considérés comme deux champs distincts, mais en fait, ils devraient se compléter les uns les autres.

«L'expérience pratique sans théorie est limitée à une situation donnée qui manque d'une approche globale et un cadre pour organiser la manière d'enseigner et d'apprendre » (Schunk $2012: 40)$.

La question comment enseigner ou apprendre une langue étrangère, était depuis longtemps au cœur des préoccupations des chercheurs dans ce domaine. Ainsi toute approche appliquée actuellement dans l'enseignement des langues trouve son inspiration dans les théories telles que le behaviorisme, le cognitivisme, le constructivisme et le socioconstructivisme. Ce sont des théories dont l'étude était centrée sur les facteurs pouvant favoriser la transmission et l'acquisition des connaissances et des savoirs. Le deuxième point qui sera développé, concernera l'évolution des méthodes de l'enseignement des langues étrangères qui ont découlé des courants de l'apprentissage cités auparavant. En ce qui concerne les méthodes, nous évoquerons les deux principales méthodes, considérées par des chercheurs comme l'application évidente des principes du courant behavioriste à savoir la méthodologie audio-orale et la méthodologie audiovisuelle. S'agissant des approches, nous nous limitons à analyser les caractéristiques de l'approche communicative et l'approche par 
compétences dont l'apport était considérable dans le domaine des langues étrangères et dont l'usage était très répandu au cours des dernières années.

\section{Le behaviorisme}

Le behaviorisme est un courant psychologique apparu aux États-Unis au début du XXème siècle. Son principe de base est l'observation des comportements de l'individu en interaction avec son environnement. Les théories d'apprentissage, fondées par Watson, Pavlov et Thorndike étaient d'une grande importance historique. Bien que ces théories diffèrent, chacune considère l'apprentissage comme un processus qui associe les « stimuli » avec les réponses. Le concept central du behaviorisme est le conditionnement. Le conditionnement classique, appelé aussi « conditionnement pavlovien » consiste à associer un stimulus neutre à un stimulus inconditionné. Selon Pavlov, le sujet n'est pas responsable de ses comportements. En se basant sur les résultats de ses recherches effectuées sur la salivation des chiens, Pavlov souligne que le sujet ne fait que réagir et cela grâce à la répétition du processus de l'association qui conduit à son tour à la construction d'un nouveau réflexe conditionné.

Les travaux de Skinner vont marquer un grand tournant dans la théorie behavioriste. La contribution majeure de Skinner est le développement du concept «le conditionnement opérant » appelé aussi, stimulus-réponse-conséquence. Il est caractérisé par la production d'un comportement volontaire en opposition au comportement involontaire produit par le conditionnement classique. Ce qui régit ce type de conditionnement est la loi de l'effet selon laquelle l'apprentissage se fait à la fois par la simultanéité et les conséquences. Dans son analyse de la triade : (Stimulus) $\rightarrow$ Réponse $\rightarrow$ Conséquence $\rightarrow$, Skinner s'est intéressé davantage au renforcement 
relié immédiatement à toute réponse. En ce sens, l'intérêt est porté sur l'aspect extérieur du comportement en rejetant toute référence aux intentions du sujet.

Cette conception behavioriste, basée essentiellement sur des conditions extérieures, a eu une influence considérable sur les projets pédagogiques des années 1970 dont le champ d'application était la pédagogie par objectifs (P.P.O). C'est une pratique éducative apparue aux Etats-Unis au cours des années 1950 et diffusée dans le domaine éducatif à travers les travaux de Bloom. Elle s'articule sur trois concepts principaux : un comportement observable, un objectif général et un objectif spécifique. En effet, selon Bloom, la réussite de l'apprentissage est centrée sur les comportements observables de l'apprenant. Avant de commencer la formation on se pose les questions suivantes : que veut-on observer à la fin de la formation? Quel comportement observable de l'apprenant montre que la formation en question a réussi ? Pour ce faire, Bloom a créé un modèle pédagogique appelé, « la taxonomie de Bloom » ou la taxonomie des objectifs. C'est un modèle qui propose une classification des niveaux d'acquisition des connaissances. C'est une classification hiérarchique des comportements cognitifs, affectifs et moteurs, établie sur la base d'un ou plusieurs critères. Parmi les principales caractéristiques de la pédagogie par objectifs, on cite la centration sur les comportements observables de l'apprenant, le rejet de toute référence à la conscience, c'est-à-dire, tout ce qui se passe dans le cerveau, et la décomposition du savoir en unités minimales.

\section{Le Cognitivisme}

Le cognitivisme est un courant psychologique constitué durant les années cinquante et qui préconise l'hypothèse selon laquelle la pensée est un processus de « traitement de l'information ». Contrairement à l'approche behavioriste dont le fondement principal repose essentiellement sur le comportement observable, l'approche cognitiviste 
s'intéresse à l'étude des stratégies mentales mises en œuvre par le sujet pour acquérir des connaissances.

Vers la fin des années cinquante, l'objet d'étude des chercheurs cognitivistes spécialisés dans le domaine des langues étrangères était de découvrir les facteurs intervenant dans ce processus d'apprentissage. Pour ce faire, la plupart des travaux de recherche, centrés sur la cognition et sa relation avec l'apprentissage des langues étrangères, se sont intéressés à l'explication de certaines hypothèses. Ortega (2014, p. 106-107) a analysé l'apport de ces hypothèses tout en résumant les différents points de vue des autres théoriciens exerçant dans le même domaine de recherche. Son travail a abouti aux conclusions suivantes :

- La théorie du traitement de l'information : Le traitement de l'information explique l'apprentissage d'une langue étrangère comme un processus de transformation progressive de la performance contrôlée. Celle-ci devient, à travers la procéduralisation, une performance automatique.

- La mémoire : L'encodage du vocabulaire de la langue étrangère dans la mémoire à long terme dépend du savoir que possède l'apprenant sur les mots de la langue cible. Il dépend aussi du contenu du vocabulaire dans la mémoire d'un bilingue. La capacité de la mémoire de travail est faible lors de l'apprentissage d'une langue étrangère. Elle évolue au fur et à mesure que le niveau de l'apprenant avance dans son apprentissage.

- L'hypothèse de l'intention : Il est possible d'apprendre une langue étrangère sans avoir l'intention de le faire, cependant on apprend beaucoup et rapidement quand on procède intentionnellement.

- L'hypothèse de l'attention : «Faire attention » dans ce contexte signifie utiliser des ressources cognitives pour traiter l'information. Ainsi, l'apprenant se 
trouvant devant une quantité d'information, il va mobiliser ses ressources cognitives pour comprendre les mots clés d'un message et négliger ceux qui n'affectent pas directement le sens.

- L'hypothèse du repérage : Cette hypothèse souligne que le repérage est un élément essentiel dans le processus de l'apprentissage des langues étrangères. A force d'observer certains aspects de la langue cible, soit en classe ou lors d'une autre expérience, l'apprenant finit par intégrer ces caractéristiques dans le système de la langue cible.

- L'hypothèse de l'interaction : Cette hypothèse soutient que l'interaction au cours de la conversation est essentielle et peut être considérée comme une condition suffisante pour l'apprentissage d'une deuxième langue. Elle insiste sur le fait qu'il faut encourager l'apprenant à produire des énoncés compréhensibles afin d'identifier leurs limites en langue étrangère et développer leur compétence langagière.

Les conclusions qu'on peut tirer des hypothèses avancées ci-dessus, est que dans le processus de l'apprentissage d'une langue étrangère, tel qu'il est analysé par les théoriciens et psychologues cognitivistes, l'apprenant est un sujet actif dans son apprentissage. Selon les cognitivistes, l'apprenant traite l'information à l'aide d'un mécanisme mental. Il apprend, intègre, réutilise les connaissances qui se construisent graduellement. Au cours de la réalisation de ce mécanisme, plusieurs facteurs (la mémoire, l'attention, l'intention et l'interaction) interviennent pour faciliter l'apprentissage.

\section{Le behaviorisme et l'enseignement des langues}

Le courant behavioriste a eu une grande influence dans le domaine de l'enseignement 
des langues étrangères surtout en Amérique du Nord, entre les années 1940 et 1970. Nelson Brooks (1964) et Robert Lado (1964) ont été deux partisans de cette perspective dont l'influence a été ressentie clairement dans le développement des matériels d'enseignement basé sur la méthodologie audio-orale, et dans la formation des enseignants (Lightbown et Spada 2013). Les activités organisées en classe mettaient l'accent sur le mimétisme et la mémorisation. Selon les adeptes de cette méthode, le développement du langage est une formation d'un ensemble d'habitudes qui, dans le cas de l'apprentissage d'une deuxième langue, vont interférer avec les nouvelles habitudes nécessaires pour la langue apprise.

Le behaviorisme était souvent lié à «l'hypothèse de l'analyse contrastive » $(\mathrm{CAH})$, qui a été développée par des linguistes structuralistes en Europe et en Amérique du Nord. Selon le «l'hypothèse de l'analyse contrastive », quand la première langue et la langue cible sont similaires, les apprenants acquièrent facilement les structures de la langue cible ; par contre, si cette dernière contient des différences, les apprenants rencontrent des difficultés. Dans le même cadre, Myles et Mitchell, (2004) soulignent que le processus de l'apprentissage d'une langue maternelle est relativement simple. On apprend un ensemble de nouvelles habitudes de la même manière par laquelle on apprend à répondre aux stimuli dans notre environnement. Le problème se pose au niveau de la langue étrangère. Les nouvelles habitudes de celle-ci interfèrent avec un ensemble de réponses bien établies dans la langue maternelle. Dans ce cas, la facilité ou la difficulté de l'apprentissage de la langue cible dépend de la similitude ou de la différence des deux langues au niveau de leur structure.

L'histoire de l'évolution des méthodes de l'enseignement des langues étrangères a été toujours marquée par le changement dans les besoins. D’abord la méthodologie traditionnelle est apparue au début du XXe siècle, formant une base dans 
la didactique des langues étrangères. Ensuite elle était remplacée par la méthode directe en mettant de côté les principes de la méthodologie traditionnelle pour un apprentissage efficace de la langue cible. Par la suite, deux méthodes, la méthode audio-orale et la méthode audio-visuelle inspirées à la fois des théories psychologiques behavioristes de l'apprentissage et de la linguistique appliquée, ont été introduites dans le domaine de l'enseignement des langues étrangères.

\section{La méthode audio-orale}

L'ancrage historique de la méthodologie audio-orale remonte à l'entrée en guerre des Etats-Unis pendant la deuxième guerre mondiale. Dans le besoin de former rapidement des militaires aux langues vivantes étrangères, l'armée américaine a fait appel à un groupe de linguistes pour l'élaboration d'un programme destiné spécialement à l'enseignement des langues. C'est à cause de l'influence de cette méthode appelée à l'époque « la méthode militaire » que vont s'élaborer les premières formes de la méthodologie audio-orale.

La linguistique appliquée était le champ d'étude interdisciplinaire tant exploité par les concepteurs de la méthode audio-orale. Ils se sont basés sur les deux axes principaux de l'analyse distributionnelle : l'axe paradigmatique et l'axe syntagmatique. Deux types de manipulation linguistique émanent de ces deux éléments à savoir la règle de la substitution, la répétition et la transformation. L'application de ces trois règles peut se faire en classe ou en laboratoire de langues. Dans un premier temps, et suivant les consignes de leur professeur, les apprenants répètent un mot ou plusieurs mots de la phrase puis procèdent à la substitution d'un mot ou d'un groupe de mots qui remplacera un terme dans la structure initiale. Ainsi cette dernière subit un changement, d'une structure simple à une structure complexe. Ces exercices structuraux portent uniquement sur la forme. 
Les objectifs de la méthode audio-orale ont été déterminés selon une distinction entre les objectifs à long terme et les objectifs à court terme. Selon Brooks (1964), les objectifs à court terme comprennent les quatre compétences langagières à savoir la compréhension de l'oral et de l'écrit, ainsi que la production de l'oral et de l'écrit. En ce qui concerne les objectifs à long terme, l'apprenant doit atteindre un niveau avancé dans l'apprentissage de la langue. Son usage de la langue doit être le même que celui d'un natif et maitriser la langue étrangère comme un vrai bilingue. Richards et Rodgers (2001, p. 56) ont envisagé les relations pédagogiques au sein de la pratique de la méthode audio-orale selon une répartition des rôles :

- Rôle de l'apprenant : Imiter l'enseignant ou la bande magnétique, réutiliser la structure en proposant de nouvelles variations paradigmatiques, mémoriser le vocabulaire à travers des dialogues simples, s'appliquer aux directives de l'enseignant, répondre aux questions de l'enseignant ;

- Rôle de l'enseignant : Employer la langue cible pour communiquer, servir de modèle de prononciation, diriger et guider le comportement langagier des apprenants, corriger la prononciation des apprenants, récompenser l'apprenant.

\section{La méthode audiovisuelle}

Les origines de la méthode audiovisuelle remontent aux années cinquante. Après la deuxième guerre mondiale, la France s'est engagée pour promouvoir l'enseignement du français langue étrangère jusqu'en faire une affaire d'Etat. Pour ce faire, le CREDIF (Centre de Recherche et d'Etude pour la Diffusion du Français) a été créé pour répondre à une demande ministérielle. Le centre CREDIF avait une double mission : faciliter l'apprentissage du français et sa diffusion à travers le monde. Les premiers travaux de ce centre de recherche ont été publiés sous forme d'un programme nommé, « le français 
fondamental ». Le public visé par ce programme concernait les élèves en situation scolaire apprenant le français langue étrangère. Le français fondamental a suscité beaucoup de critiques, on lui reprochait l'atteinte à l'intégrité de la langue française et l'inadéquation de ses programmes aux besoins langagiers des apprenants.

Les réformes menées par les méthodologues du CREDIF au milieu des années 1950, pour améliorer l'enseignement du français langue étrangère, ont abouti à l'élaboration des premières formulations de la méthode audiovisuelle. Puren (1988, p. 192) définit la méthode audio-visuelle en lui attribuant l'aspect technique suivant :

«La définition que je propose de la MAV s'appuie sur l'unique critère technique de l'intégration didactique autour du support audiovisuel. Elle exclut par conséquent les cours ne proposant que des supports sonores et/ou séparément visuels (...), et les cours dans lesquels l'emploi des supports audiovisuels n'est qu'occasionnel, pour ne retenir que ceux qui au moins permettent un emploi régulier de ces supports audiovisuels pour la présentation-explication du document de base ».

La définition de Puren met au clair l'importance des supports audiovisuels dans la méthode Structuro-globale audio-visuelle, qui a dominé en France dans les années 1960-1970. Le premier cours audiovisuel issu du programme «Voix et images de France » a été élaboré en 1962 par le CREDIF. P. Rivenc (cité par Puren, 1988), reconnait que l'influence américaine, à travers le programme le TAVOR AIDS (Teacher's Audio Visual Oral Aids) (le support audio-visuel de l'enseignant), est à l'origine de l'inspiration des méthodologues du CREDIF pour créer leur premier cours audio-visuel.

La présence de l'image et du son dans les pratiques de la méthodologie structuro-globale audio-visuelle renvoie à l'hypothèse avancée par Puren (1988), qui stipule que la méthodologie audio-orale ainsi que la méthodologie structuro-globale- 
audio-visuelle, siglée (SGAV) font partie des méthodes audio-visuelles. De plus, et dans le même contexte, Puren ajoute que l'originalité de la méthodologie SGAV réside dans le fait qu'elle représente une continuité de la méthodologie directe. Selon lui, la SGAV et MD possèdent la même organisation interne qui repose sur la simultanéité de l'application des principales méthodes de la méthodologie directe (active, directe et orale); celles-ci impliquent à leur tour d'autres méthodes complémentaires (interrogative, intuitive, imitative et répétitive).

La particularité de la méthode audio-visuelle est l'introduction du magnétophone et de petits films au sein de la classe. Ceci confère à l'apprenant un apprentissage global composé d'éléments linguistiques (grammaire, lexique, phonétique) et d'éléments extralinguistiques (le geste, le rythme, la mimique). De plus, la méthode audio-visuelle repose également sur la contextualisation de l'apprentissage à travers la présentation d'une situation de communication accompagnée des lieux et des circonstances du dialogue étudié.

\section{Critique}

Les méthodes fondées sur la théorie du behaviourisme ont subi beaucoup de critiques. Commençant par «l'hypothèse de l'analyse contrastive », un bon nombre de chercheurs ont constaté que les apprenants ne font pas toutes les erreurs anticipées par «l'hypothèse de l'analyse contrastive ». En revanche, beaucoup de leurs erreurs ne sont pas commises à cause de l'interférence de leur langue maternelle. Dans les années 1970, beaucoup de linguistes ont été convaincus que les explications avancées par le behaviorisme et «l'hypothèse de l'analyse contrastive », concernant l'acquisition d'une langue étrangère, étaient inadéquates. Ces critiques sont apparues en réaction de l'influence croissante, à l'époque, de la théorie nativiste prônée par Chomsky. Chomsky (1976) a rejeté la théorie du behaviorisme en mettant en lumière son concept de « la 
grammaire universelle »(UG). Selon lui, chaque être humain est biologiquement prédisposé pour apprendre la langue en utilisant la faculté du langage qui est responsable du stade initial du développement du langage. En se référant à cette théorie, l'apport de l'environnement seul n'est pas suffisant pour la réussite de l'apprentissage d'une deuxième langue. Les apprenants ne s'appuient pas seulement sur la répétition d'aspects mécaniques du langage mais font usage de leur esprit qui contient du langage (Myles \&Mitchell, 2004). White (2004) et d'autres linguistes ont soutenu l'idée que la théorie de « la grammaire universelle » établie par Chomsky, offre les meilleures perspectives pour mieux comprendre le processus de l'apprentissage d'une langue étrangère.

Deuxièmement, la méthode audio-orale n'a pas répondu aux attentes de plusieurs praticiens de l'époque. On lui reproche des lacunes au niveau de sa conceptualisation du processus d'apprentissage. Chomsky a rejeté les fondements de l'approche structuraliste ainsi que la conception de l'apprentissage par la théorie behavioriste. Selon Chomsky (1966, p. 153) : «La langue n'est pas une structure habituelle. Un comportement linguistique ordinaire implique typiquement l'innovation, la formulation de nouvelles phrases et nouveaux exemples conformément aux règles de l'abstraction et complexité ».

Finalement, malgré le succès réalisé par la méthode audiovisuelle, elle était sujet de plusieurs contestations. On a critiqué la surcharge de son dispositif d'enseignement qui exige des conditions spécifiques : nombre limité d'apprenants, des cours intensifs, formation étalée sur une longue durée, formation spécifique des enseignants et enfin la cherté du matériel d'enseignement (ex. magnétophone/ laboratoire de langue). Vers la fin des années soixante, la méthodologie audiovisuelle est entrée en déclin surtout avec l'émergence d'autres théories linguistiques comme le fonctionnalisme et d'autres 
d'ordre psychologiques inspirées du courant cognitiviste. Ainsi les réflexions des théories cognitivistes sur l'apprentissage des langues étrangères ont été un champ favorable pour donner naissance à des approches, telle que l'approche communicative dont l'application en classe de langues étrangères remplacera la méthodologie audioorale et la méthodologie audio-visuelle.

\section{L'approche communicative}

Deux changements majeurs de nature linguistique ont été à l'origine de l'avènement de l'approche communicative. D’abord, vers la fin des années 1960, deux méthodes ont été remises en question, la méthodologie situationnelle anglaise en grande Bretagne et la méthodologie audiovisuelle aux Etats-Unis. On reprochait à ces deux méthodes la centration de l'apprentissage des langues étrangères sur la forme et la structure au détriment du contexte. Ensuite, les travaux de Noam Chomsky, couronnés par sa théorie de « la grammaire universelle », avaient contribué aussi à l'élaboration des premiers fondements de l'approche communicative. Les premiers débuts des théories cognitivistes ont été initiés par Chomsky qui stipule que chaque être humain, dispose d'une capacité innée à déchiffrer et à comprendre un code langagier grâce à un mécanisme mental spécifique. Mais le déclenchement réel de l'approche communicative a été avec la décision du «Conseil de l'Europe » d'instaurer le « Niveau seuil » en 1975, inspiré du modèle américain le « Threshold Level », pour l'enseignement des langues étrangères. Cette décision est venue en réaction à la nécessité d'enseigner aux adultes les principales langues du « Marché Commun Européen ».

En 1971, un groupe d'experts a commencé à étudier la possibilité de développer des cours de langue. Le groupe s'est servi des études sur les besoins des apprenants en langues européennes, et le premier document a été préparé par un linguiste britannique, 
David Wilkins (1972), qui proposait une définition fonctionnelle ou communicative du langage pouvant servir de base à l'élaboration des programmes relatifs à l'enseignement des langues. La contribution de Wilkins était d'analyser le sens de la communication au lieu de décrire le noyau du langage à travers les concepts traditionnels de la grammaire et du vocabulaire. Selon lui, la communication est dotée de deux catégories : la catégorie notionnelle (le temps, la séquence, la quantité, le lieu et la fréquence) et la catégorie fonctionnelle (demandes, refus, offres, plaintes).

L'appellation de l'approche fonctionnelle-notionnelle a été employée pour désigner l'approche communicative dont le but principal était de développer la compétence communicative chez l'apprenant (Hymes, 1972). D'après Hymes, l'acquisition d'une compétence communicative offre à l'apprenant un savoir sur l'usage la langue. Quatre dimensions de la compétence communicative ont été identifiées par Canale et Swain (1980) : La compétence grammaticale (la grammaire et le lexique), la compétence sociolinguistique (comprendre le contexte social de la communication), la compétence discursive (l'interprétation des éléments d'un message) et la compétence stratégique (savoir initier, terminer, maintenir, réparer et réorienter la communication). Sur le plan de l'apprentissage, l'approche communicative privilégie les activités basées sur des situations de communication réelles qui garantissent un apprentissage significatif au lieu de se focaliser uniquement sur la description linguistique de la langue.

Concernant l'élaboration des programmes, hormis la contribution de David Wilkins, le conseil de l'Europe a procédé à un changement dans le syllabus en incluant la description des objectifs déterminés pour les cours des langues étrangères, les situations de communication nécessaires pour l'utilisation de la langue étrangère (ex. voyage, travail, faire les courses), des sujets dont ils souhaitent parler (informations 
personnelles, éducation, les courses), le vocabulaire et la grammaire nécessaire. Cidessous est un exemple de modèles des syllabus décrit par Yalden (1983) :

(1) Structures et fonctions : Wilkins (1976)

(2) Structural, fonctionnel, instrumental : Allen (1980)

(3) Notionnel : Wilkins (1976)

(4) Interactionnel : Widdowson (1979)

S'agissant des activités, Littlewood (1981) fait la distinction entre «activités de communication fonctionnelle » et « activités d'interaction sociale » comme principaux types d'activités dans l'approche communicative. Les activités de communication fonctionnelle comprennent des tâches telles que l'observation des similitudes et des différences dans un ensemble des images; découvrir des éléments manquants dans une carte ou une image ; communiquer des instructions derrière un écran à un autre apprenant ; résoudre les problèmes à partir d'indices partagés. Les activités d'interaction sociale incluent des sessions de conversation et de discussion, des dialogues et des jeux de rôle, des simulations, des sketchs et des débats.

Dans le cadre de l'approche communicative adoptée l'enseignant remplit plusieurs fonctions. Selon la description de Breen et Candlin (1980), l'enseignant a deux rôles principaux. D'abord un facilitateur du processus de la communication entre tous les participants en classe, et entre ces participants et les différentes activités. Ensuite il joue le rôle d'un participant indépendant au sein du groupe d'apprentissageenseignement. Ces deux fonctions impliquent un ensemble de rôles secondaires pour l'enseignant : un organisateur de ressources, un détenteur du savoir et un meneur des activités en classe (Jack Richards et Theodore Rodgers, 2001). Un troisième rôle pour l'enseignant est celui d'un chercheur et d'un apprenant, avec sa contribution en termes de connaissances, de compétences et d'expériences vécues (Breen et Candlin, 1980). 
Dans le but de s'adapter aux propositions didactiques dictées par l'approche communicative, beaucoup de manuels ont été réalisés pour orienter l'enseignement des langues étrangères. Il s'agit de proposer aux apprenants des tâches d'apprentissage communicatives telles que les jeux de rôle et des simulations intégrées dans les leçons. Les documents proposés sont caractérisés par l'authenticité ; ce qui permet à l'élève d'apprendre la langue étrangère dans un contexte proche de la réalité. Cela peut inclure des documents tirés de la vie réelle comme des affiches de tout type, des publicités, des journaux et des sources graphiques (image, carte, symbole). Une leçon typique se déroule autour d'un seul thème, une analyse des tâches pour le développement du thème, une description de la situation, des questions de compréhension et des exercices de paraphrase. S'ajoute à cela, une variété d'activités de communication, de simulations basées sur les tâches sont préparées pour accompagner et compléter la leçon (Richards et Rodgers, 2001).

En conclusion, trois principes de base caractérisent l'approche communicative. Le premier principe concerne la centration sur l'apprenant ; celui-ci est actif dans son apprentissage, et prend en charge son apprentissage d'une manière autonome ; ses besoins sont identifiés et analysés pour envisager un enseignement progressif. Le deuxième principe est en relation avec l'interaction et la priorité donnée à la communication comme un moyen facilitateur de l'apprentissage des langues étrangères. Cela renforce la dynamique du groupe où les participants interagissent dans une atmosphère de confiance qui valorise l'échange et l'entraide. Le troisième principe est relatif à l'authenticité des documents qui vise à rendre l'apprenant opérationnel dans des situations de communication réelles à travers les programmes des manuels ainsi que les activités proposées tout au long du processus de l'apprentissage. Malgré les apports de l'approche communicative dans l'évolution des méthodes de l'enseignement des 
langues étrangères, elle a subi, elle aussi, le même sort que celui des méthodes précédentes. La plupart des critiques reprochent à l'approche communicative l'absence de la langue écrite et de la grammaire dans le processus de l'apprentissage des langues étrangères. Pour encourager l'interaction entre les apprenants, la langue orale était prédominante au dépend de l'écrit, de même pour la grammaire, devenue quasi inexistante à cause de la volonté de tout étudier en contexte. S'ajoute à cela, et d'après certains partisans de l'approche communicative, celle-ci, à l'instar des autres méthodes n'a pas pu réaliser ses objectifs préalablement déterminés, à cause des contraintes qui relèvent du milieu scolaire ou de l'environnement socioculturel de l'apprenant.

\section{Le constructivisme}

Le constructivisme est une théorie de l'apprentissage développée par le psychologue suisse Piaget en 1964. C'est une théorie qui valorise davantage le rôle de l'apprenant dans la construction de son savoir. La théorie de l'apprentissage prônée par le constructivisme puise ses origines dans les travaux de Jean Piaget. Le psychologue stipule que l'acte d'apprendre relève de l'acte de construire et l'acquisition de connaissance se réalise à travers « l'assimilation » et « l'accommodation ». Ce sont deux mécanismes indissociables à l'activité mentale. Piaget (1977, pp. 124-125) donne l'explication suivante :

« Par le fait même que l'assimilation et l'accommodation vont toujours de pair, le monde extérieur ni le moi ne sont jamais connus indépendamment l'un de l'autre : le milieu est assimilé à l'activité du sujet en même temps que celle-ci s'accommode à celui-là. En d'autres termes, c'est par une construction progressive que les notions du monde physique et du moi intérieur vont s'élaborer en fonction l'un de l'autre et les processus d'assimilation et d'accommodation ne sont que les instruments de cette construction, sans jamais représenter le résultat lui-même». 
Pendant le processus de l'apprentissage, l'apprenant réintègre des éléments externes à l'intérieur d'une structure interne préétablie ce qui donne lieu à une assimilation ; dans le cas contraire, l'organisme s'adapte aux variations externes par le biais de l'accommodation. De ce fait, l'apprenant interagit avec son environnement et devient actif dans la construction de ses connaissances. Conformément à la conception de Piaget, il ne s'agit pas d'accumuler ou d'acquérir un savoir, mais au contraire de le construire.

Pour une meilleure application d'une pédagogie constructiviste, Doolittle (1999) propose un ensemble de conditions : Offrir aux apprenants des situations d'apprentissage proches de leur vécu quotidien ; encourager l'interaction et la coopération entre les apprenants ; présenter des situations d'apprentissage porteuses de sens ; fonder l'apprentissage sur les acquis des apprenants ; privilégier l'évaluation formative continue des apprenants ; solliciter l'autorégulation continue des apprenants ; guider et faciliter l'apprentissage des apprenants ; réviser les contenus et les présenter selon les besoins des apprenants. Dans le même contexte, Brahimi, Farley, et Joubert (2011) évoquent d'autres stratégies pédagogiques préconisées par le modèle constructiviste telles que les expérimentations, la réalisation de projets, la résolution de problèmes globaux et significatifs comme l'apprentissage par problèmes, les simulations, l'apprentissage collaboratif (ex : communauté de pratique, communauté d'apprentissage ou technique d'enseignement réciproque) et l'accompagnement cognitif (ex: coaching, mentorat, tutorat, supervision, etc.).

Ainsi et compte tenu de ces fondements, le constructivisme est un modèle pédagogique qui rend l'apprenant responsable de son apprentissage, c'est lui qui gère ses propres démarches sous la supervision et l'accompagnement de l'enseignant. De ce fait «l'approche par compétences » est considérée comme l'une des applications les 
plus connues du constructivisme et dont la concrétisation a pris lieu d'abord dans le secteur industriel avant d'atteindre celui de l'éducation.

\section{L'approche par compétences}

Les prémices de «l'approche par compétences » siglée (L'APC) ont débuté d'abord aux Etats-Unis dans le secteur industriel et en particulier les formations professionnelles. Perfectionner les compétences du personnel et améliorer leur productivité étaient l'objectif principal des tenants de l'approche par compétences. Puis, vers la fin des années 1960, et en réaction contre les méthodes centrées soit sur l'apprenant, soit sur l'enseignant, le matériel ou le contenu, l'approche par compétences s'est imposée dans le domaine de l'éducation en adoptant un nouveau cadre conceptuel basé sur la notion de la compétence. Ensuite, et toujours dans la même optique de repenser leur système éducatif, certains pays européens ont adopté l'approche par compétences qui va remplacer la pédagogie par objectifs ; approche qui a dominé dans le monde scolaire tout au long du XXème siècle. Le concept « compétence » a connu une multitude de définitions à partir desquelles émergent d'autres concepts importants qui constituent les principales composantes de l'approche par compétences. Les chercheurs font des recours fréquents à des mots comme : Savoir, ressources, capacité, connaissance, situation, fonction, activité, action. Partant de ce constat, les actions entreprises par le sujet représentent le noyau de l'acte de l'apprentissage. Celui-ci doit mobiliser un ensemble de ressources (le savoir, le savoir-faire, le savoir-être) pour pouvoir agir devant une situation complexe.

L'approche par compétences a eu une grande influence dans le domaine de l'éducation depuis son émergence dans les années 1970. Les premiers travaux de recherche en rapport avec cette approche ont été effectués par De Ketele et Gerard. Selon eux (2005, p. 188), l’approche par compétences « Cherche à développer la 
possibilité par les apprenants de mobiliser un ensemble intégré de ressources pour résoudre une situation-problème appartenant à une famille de situations ». On remarque que cette définition rejoint les définitions des autres chercheurs citées auparavant pour mettre en évidence les grandes lignes directives de cette approche, et dont l'intérêt primordial est la centration sur le profil de sortie de l'apprenant tout en évaluant les compétences acquises à la fin de chaque phase d'apprentissage. D’autres spécialistes comme Docking (1994), estiment que l'évaluation axée sur les compétences acquises et sur la spécification des résultats attendus, contribue à l'amélioration de la qualité de l'enseignement et de l'apprentissage des apprenants. Docking poursuit également, qu'aux Etats-Unis, ces effets bénéfiques ont été observés dans tous les niveaux et types d'éducation et de formation, de l'école primaire à l'université et aussi dans les centres de formation académique et professionnelle.

Selon Richards et Rodgers (2001), la conception de l'apprentissage comme processus basé sur la démonstration du savoir plutôt que sur le savoir lui-même, confère à l'approche par compétences le privilège de se distinguer par les caractéristiques suivantes : La centration sur l'apprenant, l'apprentissage doit cibler la réalisation d'une tâche à la fin de l'année scolaire, offrir un apprentissage significatif et expliquer son utilité à l'apprenant et l'évaluation du savoir agir de l'apprenant.

L'approche par compétences n'est pas conçue autour de la notion du savoir mais autour de celle de la compétence. L'objectif visé par cette approche est de tester ce que l'apprenant peut accomplir avec la langue et non son savoir sur la langue. La centration mise sur les compétences acquises ou les résultats d'apprentissage exige une planification spécifique du programme, des stratégies d'enseignement et d'évaluation adaptées aux objectifs prédéterminés dès le début de l'acte d'apprentissage. C'est-àdire, au lieu d'une évaluation dictée par les normes, les apprenants seront évalués en 
fonction de leur capacité à effectuer des tâches d'apprentissage spécifiques (Docking 1994).

Partant de ce principe, L'APC est basée sur la perspective fonctionnelle et interactionnelle de la langue. Son but est d'enseigner la langue en relation avec le contexte social dans lequel elle est utilisée ; et par conséquent développer une compétence communicative chez l'apprenant. Hymes (1972) a défini la compétence de communication comme : «la connaissance des règles psychologiques, culturelles et sociales qui commandent l'utilisation de la parole dans un cadre social ». A ce sujet, Richards et Rodgers (2001), stipulent que l'approche par compétences est le meilleur cadre de référence pour un enseignement des langues basé sur des situations similaires de celles de la vie quotidienne et dont les compétences langagières requises peuvent être déterminées préalablement. Cela suppose que pendant la préparation des curriculums, les didacticiens doivent anticiper voire même prédire le vocabulaire et les structures relatives aux situations proches du vécu de l'apprenant et les organiser selon une répartition séquentielle. Malgré la liberté accordée aux enseignants de développer leurs propres stratégies et techniques au sein de leur classe de langue, les cours sont développés autour des compétences à acquérir plutôt d'être organisés autour de thèmes linguistiques spécifiques. Les activités sont conçues selon une répartition modulaire et favorisent la manifestation de la performance des apprenants pour une meilleure pratique des compétences requises.

\section{Conclusion}

Au terme de cette étude, et après avoir passé en revue les principaux courants de l'apprentissage (behaviorisme, cognitivisme, constructivisme) ainsi que les méthodes mises en application dans le domaine éducatif et en particulier dans l'enseignement des langues étrangères, on peut déduire que ce dernier était souvent dicté par des besoins 
catégorisés selon le public visé dans un pays donné. Les besoins varient selon la volonté du cadre institutionnel structurant l'apprentissage en question (diffusion de la langue, nécessité politique ou économique, échange culturel, coopération éducative etc.). Les méthodes et les approches appliquées pour réaliser cet objectif ont connu une succession chronologique caractérisée par des chevauchements, des retours en arrière ou des adaptations. Cependant, l'objectif primordial de la plupart des applications méthodologiques dans l'enseignement des langues étrangères est de doter l'apprenant d'une compétence communicative basée sur l'acquisition du trio : Savoir/savoirfaire/savoir-être à la fin d'un processus d'apprentissage. Pour ce faire, les didacticiens, dans leurs travaux d'élaboration d'une méthode ou d'une approche, procèdent par une sorte de centration soit sur l'aspect linguistique de la langue ou le savoir sur la langue ; soit sur l'aspect fonctionnel de la langue qui permet à l'apprenant d'être opérationnel dans une situation de communication donnée ; soit sur l'ensemble des compétences qu'un apprenant doit acquérir (compétences linguistique, sociolinguistique, et pragmatique).

Elle n'existe pas une seule et unique méthode pour enseigner une langue étrangère. De surcroît on ne peut pas garantir un enseignement homogène pour tous les apprenants. Ces derniers peuvent être différents au niveau de leurs aptitudes linguistiques et leurs stratégies d'apprentissage. Les enseignants sont donc incités à se familiariser avec les théories d'apprentissage et les méthodes d'enseignement et choisir les plus convenables selon le but de l'activité langagière, les besoins des apprenants, leurs attitudes envers la méthode adoptée, et le contexte socioculturel. 


\section{Références}

Allen, J. P. B. (1980). A three-level curriculum model for second language education. Keynote address, Ontario Modern Language Teachers Association, Toronto, Ontario

Brahimi, C., Farley, C., \& Joubert, P. (2011). L'approche par compétences : un levier de changement des pratiques en santé publique au Québec. Québec : Institut national de santé publique du Québec.

Breen, M. \& Candlin, C. (1980). The essentials of a communicative curriculum in language teaching. Applied Linguistics, 1 (2), 89-112.

Brooks, N. (1964). Language and language learning : Theory and Practice. 2nd Edition. New York : Harcourt, Brace, \& World.

Canale, M., \& Swain, M. (1980). Theoretical bases of communicative approaches to second language teaching and testing. Applied linguistics, 1(1), 1-47.

Chomsky, N. (1966). Current issues in linguistic theory. Mouton : The Hague

Chomsky, N. (1976). Reflections on Language. Pantheon, New York,

De Ketele, J. M., \& Gerard, F. M. (2005). La validation des épreuves d'évaluation selon l'approche par les compétences. Mesure et Évaluation En Éducation, 28 (3), 126.

Docking, R. (1994). Competency-based curricula-the big picture. Prospect, 9 (2), 8-17.

Doolittle, P. E., \& Camp, W. G. (1999). Constructivism : The career and technical education perspective. Journal of Vocational and Technical Education, 16 (1), 23-46.

Hymes, D. (1972). On Communicative Competence. Dans J. Pride, \& J. Holmes (dir.), Sociolinguistics (p. 269-285). Harmondsworth Penguin Books.

Lado, R. (1964). Language teaching, a scientific approach. New York : McGraw-Hill Lightbown, P. M., \& Spada, N. (2013). How languages are learned 4th edition-Oxford Handbooks for Language Teachers. Oxford : Oxford university press.

Littlewood, W. (1981). Communicative language teaching. Cambridge : Cambridge University Press.

Myles, F., \& Mitchell, R. (2007). Using information technology to support empirical SLA research. Journal of Applied Linguistics and Professional Practice, 1 (2), 169-196.

Ortega, L. (2014). Understanding second language acquisition. Routledge. 
Piaget, J. (1977). La naissance de l'intelligence chez l'enfant (Vol. 370). Paris : Delachaux et Niestlé.

Puren, C. (1988). Histoire des méthodologies de l'enseignement des langues. CLE international

Richards, J., \& Rodgers, T. (2001). Approaches and methods in language teaching. New York : Cambridge University Press.

Schunk, D. H. (2012). Learning theories : An educational perspective. Pearson.

White, L. (2003). Second language acquisition and universal grammar. Cambridge University Press.

Widdowson, H.G. (1979). Explorations in applied linguistics. Oxford : Oxford University Press.

Wilkins, D. (1972). Linguistics in language teaching. Cambridge : MFT Press

Wilkins, D. (1976). Notional syllabuses. Oxford : Oxford University Press.

Yalden, J. (1983). The communicative syllabus : Evolution, design and implementation. Oxford : Pergamon Press. 